\title{
Semirelativistic Constituent-Quark Model with Goldstone-Boson-Exchange Hyperfine Interactions
}

\author{
R.F. Wagenbrunn ${ }^{1}$, L.Ya. Glozman ${ }^{2}$, W. Plessas ${ }^{1}$, K. Varga ${ }^{3}$ \\ ${ }^{1}$ Institute for Theoretical Physics, University of Graz, Universitätsplece 5, \\ A-8010 Graz, Austria \\ ${ }^{2}$ Institute for Theoretical Physics, University of Tübingen, Auf der Morgen- \\ stelle 14, D-72076 Tübingen, Germany \\ ${ }^{3}$ Theory Division, Argonne National Laboratory, Argonne, IL 60439, USA
}

\begin{abstract}
We address the semirelativistic constituent-quark model with linear confinement and a hyperfine interaction deduced from Goldstone-boson exchange. In the version where the latter is represented by pseudoscalar meson exchange $\left(\pi, K, \eta, \eta^{\prime}\right)$ and restricted to the spin-spin component only a unified description of all light- and strange-baryon spectra is provided in close agreement with phenomenology. We discuss the extension of the model that includes also the tensor components of the pseudoscalar meson exchange and in addition vector-meson $\left(\rho, \omega, K^{*}, \phi\right)$ and scalar-meson $(\sigma)$ exchanges. A preliminary version thereof provides a description of the baryon spectra with similar good quality.
\end{abstract}

The spontaneous breaking of chiral symmetry in the $u, d, s$ sector of QCD indicates that the relevant effective degrees of freedom for light and strange baryons at low energies are furnished by constituent quarks and Goldstone bosons, while the original QCD degrees of freedom, current quarks and gluons, are no longer active. The constituent quarks are to be viewed as quasiparticles with an acquired dynamical mass and a finite extension; the Goldstone bosons are manifested by the pseudoscalar mesons. Consequently, baryons should be considered as systems of three constituent quarks interacting by Goldstoneboson exchange and being subject to confinement [1].

In this spirit we developed a semirelativistic constituent-quark model $[2,3]$ based on the following Hamiltonian:

$$
H=\sum_{i=1}^{3} \sqrt{{p_{i}{ }^{2}+m_{i}^{2}}}+V_{\chi}+V_{\text {conf }} .
$$




\section{DISCLAIMER}

This report was prepared as an account of work sponsored by an agency of the United States Government. Neither the United States Government nor any agency thereof, nor any of their employees, make any warranty, express or implied, or assumes any legal liability or responsibility for the accuracy, completeness, or usefulness of any information, apparatus, product, or process disclosed, or represents that its use would not infringe privately owned rights. Reference herein to any specific commercial product, process, or service by trade name, trademark, manufacturer, or otherwise does not necessarily constitute or imply its endorsement, recommendation, or favoring by the United States Government or any agency thereof. The views and opinions of authors expressed herein do not necessarily state or reflect those of the United States Government or any agency thereof. 


\section{DISCLAIMER}

Portions of this document may be illegible in electronic image products. Images are produced from the best available original document. 
Here, $\boldsymbol{p}_{i}$ are the 3-momenta and $m_{i}$ the masses of the constituent quarks. The relativistic form of the kinetic energy allows to avoid disturbing shortcomings of nonrelativistic quark models (see, e.g., the discussion in Ref. [2]). The confinement potential $V_{\text {conf }}$ is taken in linear form. The chiral potential $V_{x}$ consists of the (most important) spin-spin interaction provided by the exchange of the pseudoscalar octet and singlet mesons. For further details and explanations see Ref. [3].
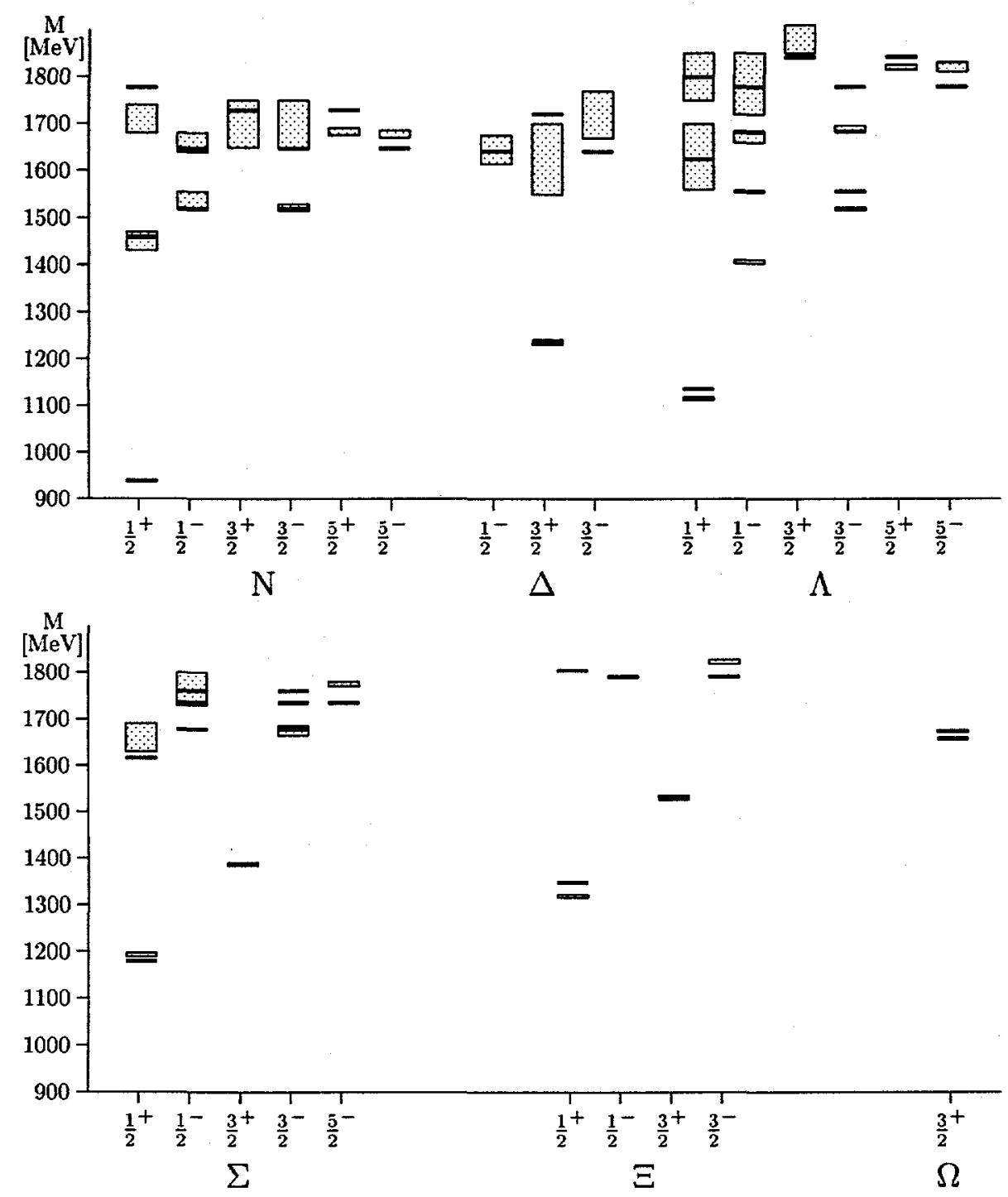

Figure 1. Energy levels of the lowest light- and strange-baryon states with total angular momentum and parity $J^{P}$ for the model of Ref. [3]. 
In Fig. 1 we show the spectra of the light and strange baryons produced by this version of the chiral constituent quark model. One observes a rather satisfactory agreement with the phenomenological energy levels as given by the PDG [4]. In particular, the correct level orderings of positive- and negativeparity excitations are provided by the hyperfine interaction $V_{x}$. Due to its specific flavor dependence this is achieved simultaneously in the light and strange sectors. Thereby a severe shortcoming of previous constituent quark models
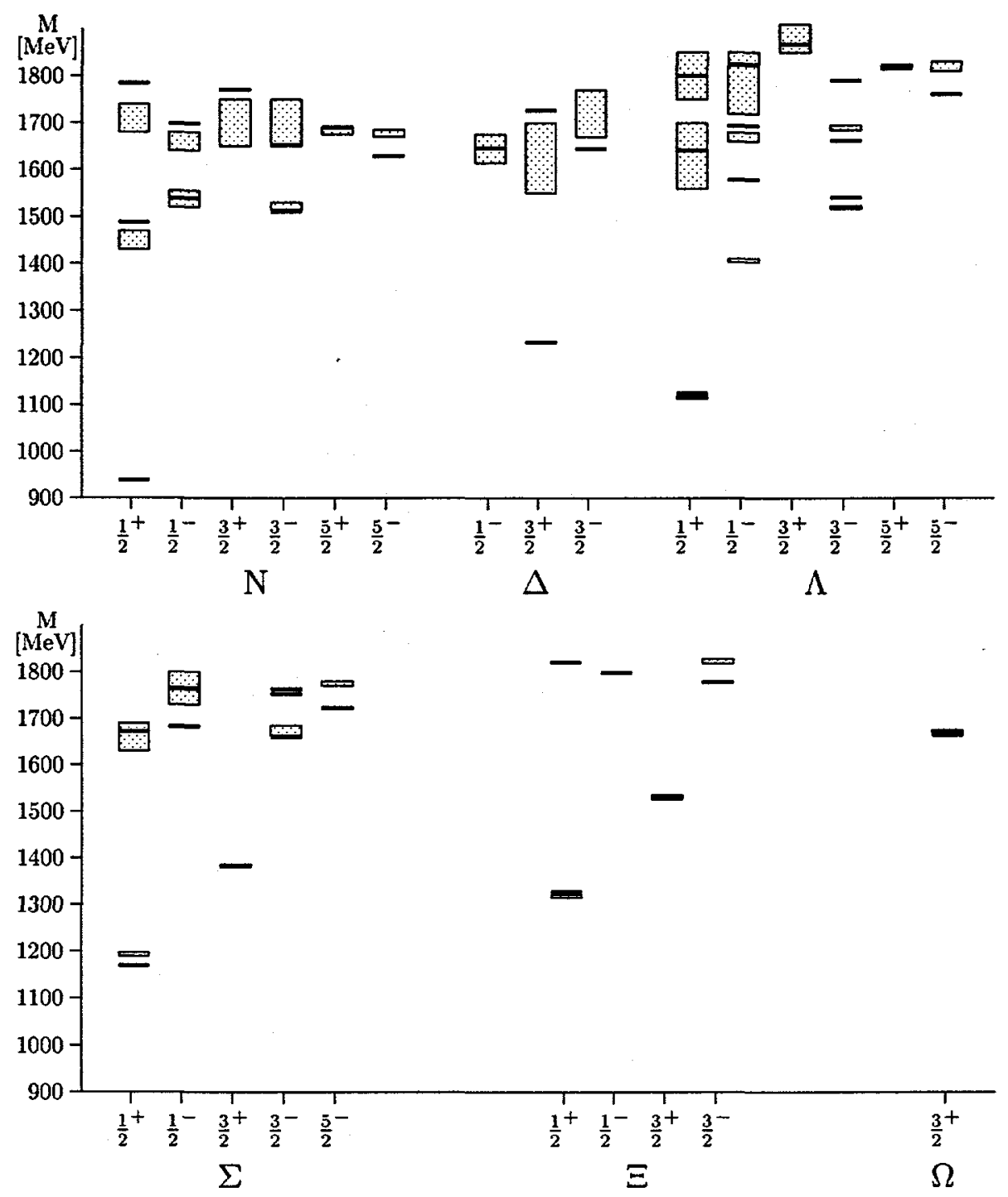

Figure 2. As in Fig. 1 but for a preliminary version of an extended model with the full interactions from pseudoscalar-, vector-, and scalar-meson exchanges included. 
relying mostly on a one-gluon exchange hyperfine interaction is remedied.

In order to account for the further details in the hyperfine structure of the baryon levels the chiral interaction must be extended beyond the spin-spin component, i.e., to include the full potential of the pseudoscalar meson exchange. At the same time it is advised to consider multiple Goldstone-boson exchanges. In this regard we introduce vector $\left(\rho, \omega, K^{*}, \phi\right)$ and scalar $(\sigma)$ meson exchanges. Thus, we end up with an extended chiral quark model whose chiral hyperfine interaction includes all possible central, spin-spin, tensor, and spin-orbit components of the pseudoscalar, vector, and scalar mesons. In a preliminary version thereof we have fixed all meson masses to their experimental values and kept all meson-quark coupling constants at the magnitudes as deduced from meson-nucleon phenomenology. In this way we have been able to avoid a proliferation of open parameters. In fact, only a few free parameters are needed to produce a spectrum as, for example, shown in Fig. 2.

The extended model meets further requirements of the baryon spectra. While the spin-spin components of the pseudoscalar- and vector-meson exchange interactions have the same sign for the short-distance interactions (providing the proper level orderings in the baryon spectra), the corresponding tensor components have opposite signs and largely cancel each other. The net tensor-force effects are thus small. This behavior is just required by the baryon spectra as the observed splittings within $L S$ multiplets (like, e.g., $N(1535)$ $N(1520)$ ) are also small. Similar to the tensor-force contributions a partial cancellation of the spin-orbit effects from different meson exchanges occurs in the extended model.

At present we are still facing the problem of a proper description of the $\Lambda(1405)$ state. We expect this state to be affected to a large extent by higher Fock components other than just $\{Q Q Q\}$. A downshift of this particular state, which lies quite close to the $N \bar{K}$ threshold, by including mesonic components has already been observed, e.g., in Ref. [5]. It is one of our future aims to extend the chiral constituent-quark model to include $\{Q Q Q$ meson $\}$ states. This would constitute a natural and consistent generalization of the model and at the same time allow for a more realistic description of baryon resonances with finite widths.

Acknowledgement. R.F.W. wants to thank the ECT* and the Paul-Urban Foundation for financial support.

\section{References}

1. L.Ya. Glozman and D.O. Riska: Phys. Rep. 268, 263 (1996)

2. L.Ya. Glozman, Z. Papp, W. Plessas, K. Varga, R. F. Wagenbrunn: Phys. Rev. C 57, 3406 (1998)

3. L.Ya. Glozman, W. Plessas, K. Varga, R. F. Wagenbrunn: Preprint hepph/9706507, Phys. Rev. D (to appear)

4. Particle Data Group, C. Caso et al.: Eur. Phys. J. C 3, 1 (1998) 
5. M. Arima, S. Matsui, K. Shimizu: Phys. Rev. C 49, 2831 (1994) 